\title{
The brace helices of MLKL mediate interdomain communication and oligomerisation to regulate cell death by necroptosis
}

\author{
Katherine A. Davies ${ }^{1,2} \cdot$ Maria C. Tanzer ${ }^{1,2} \cdot$ Michael D. W. Griffin ${ }^{3} \cdot$ Yee Foong Mok ${ }^{3}$ Samuel N. Young ${ }^{1}$ Rui Qin ${ }^{1,4}$. \\ Emma J. Petrie ${ }^{1,2} \cdot$ Peter E. Czabotar ${ }^{1,2} \cdot$ John Silke $\mathbb{D}^{1,2} \cdot$ James M. Murphy ${ }^{1,2}$
}

Received: 12 November 2017 / Revised: 4 January 2018 / Accepted: 8 January 2018 / Published online: 14 February 2018

(c) ADMC Associazione Differenziamento e Morte Cellulare 2018

\begin{abstract}
The programmed cell death pathway, necroptosis, relies on the pseudokinase, Mixed Lineage Kinase domain-Like (MLKL), for cellular execution downstream of death receptor or Toll-like receptor ligation. Receptor-interacting protein kinase-3 (RIPK3)-mediated phosphorylation of MLKL's pseudokinase domain leads to MLKL switching from an inert to activated state, where exposure of the N-terminal four-helix bundle (4HB) 'executioner' domain leads to cell death. The precise molecular details of MLKL activation, including the stoichiometry of oligomer assemblies, mechanisms of membrane translocation and permeabilisation, remain a matter of debate. Here, we dissect the function of the two 'brace' helices that connect the 4HB to the pseudokinase domain of MLKL. In addition to establishing that the integrity of the second brace helix is crucial for the assembly of mouse MLKL homotrimers and cell death, we implicate the brace helices as a device to communicate pseudokinase domain phosphorylation event(s) to the N-terminal executioner 4HB domain. Using mouse: human MLKL chimeras, we defined the first brace helix and adjacent loop as key elements of the molecular switch mechanism that relay pseudokinase domain phosphorylation to the activation of the 4HB domain killing activity. In addition, our chimera data revealed the importance of the pseudokinase domain in conferring host specificity on MLKL killing function, where fusion of the mouse pseudokinase domain converted the human $4 \mathrm{HB}+$ brace from inactive to a constitutive killer of mouse fibroblasts. These findings illustrate that the brace helices play an active role in MLKL regulation, rather than simply acting as a tether between the 4HB and pseudokinase domains.
\end{abstract}

Edited by E. Baehrecke

These authors contributed equally: Katherine A. Davies, Maria C. Tanzer.

Joint senior authors: John Silke, James M. Murphy.

Electronic supplementary material The online version of this article (https://doi.org/10.1038/s41418-018-0061-3) contains supplementary material, which is available to authorised users.

$\triangle$ John Silke

silke@wehi.edu.au

$\triangle$ James M. Murphy

jamesm@wehi.edu.au

1 The Walter \& Eliza Hall Institute of Medical Research, Parkville, VIC, Australia

2 Department of Medical Biology, The University of Melbourne, Parkville, VIC, Australia

3 Department of Biochemistry \& Molecular Biology, The University of Melbourne, The Bio21 Institute, Parkville, VIC, Australia

4 School of Pharmaceutical Sciences, Tsinghua University, Beijing, China

\section{Introduction}

Mixed Lineage Kinase domain-Like protein (MLKL) is the most terminal known obligate effector of the programmed cell death pathway necroptosis $[1,2,3,4]$. Necroptosis is an inflammatory form of cell death, in which the plasma membrane becomes permeabilised, releasing damageassociated molecular patterns (DAMPs) to alert the innate immune system [5, 6, 7, 8]. It has been implicated in tissue damage associated with a range of human pathologies, including ischaemic reperfusion injuries [9, 10, 11, 12], and diseases of the liver $[13,14,15]$. Death receptor and Tolllike receptor (TLR) -3 and -4 ligation are known to trigger necroptosis, however the best studied pathway is death induction via TNF signalling. In certain cellular contexts, TNF signalling can activate a kinase cascade in which (auto)phosphorylated RIPK1 forms a functional amyloid oligomer with RIPK3 [16, 17], which can then activate MLKL by phosphorylation $[1,2,4,18,19,20]$. This leads to a conversion of MLKL from an inert cytoplasmic 
a
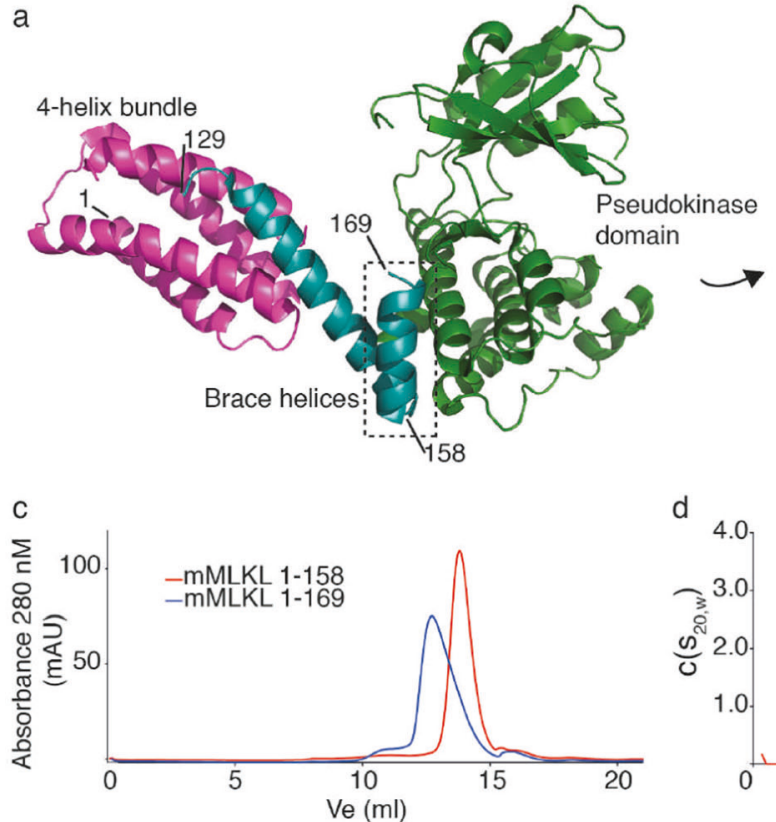

g

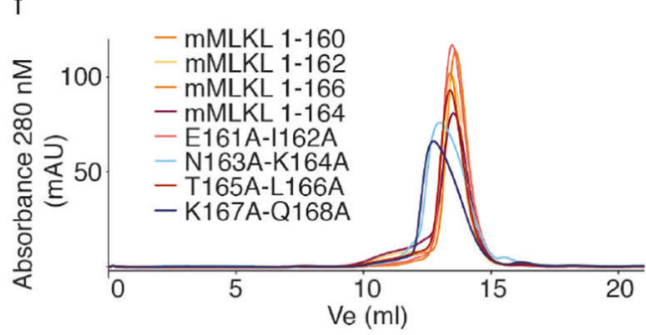

d

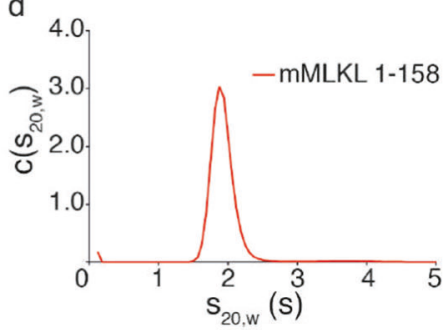

b

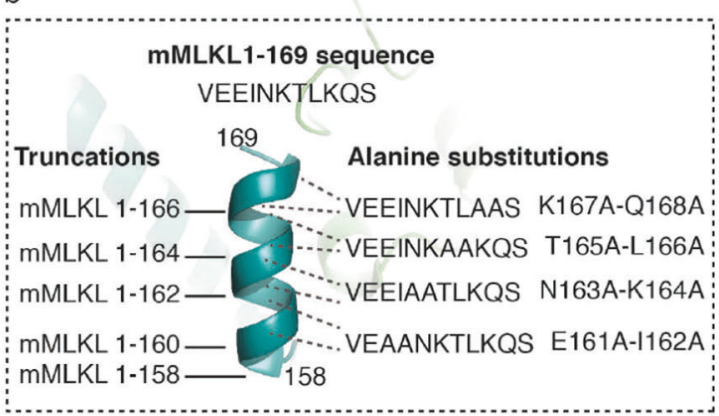

e
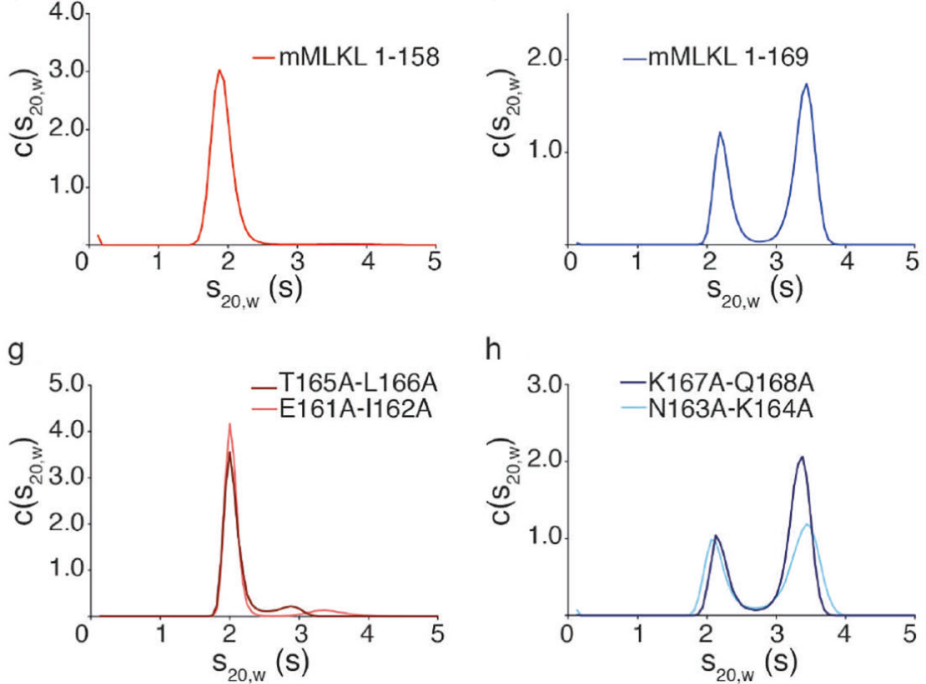

h

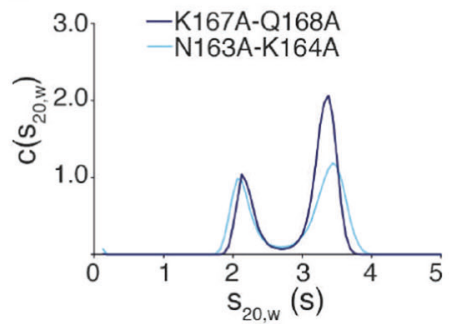

Fig. 1 Residues 159-169 of the second brace helix are responsible for trimerisation of mMLKL 1-169 in vitro. a The structure of full-length mouse MLKL (PDB:4BTF) [1]. The 4HB is coloured magenta, the PsK domain green and the two-helix brace in teal. $\mathbf{b}$ Brace truncations and mutations are highlighted on the second brace helix. c Overlay of analytical gel filtration of mMLKL 1-158 and 1-169. d, e Standardised continuous sedimentation coefficient $\left[\mathrm{c}\left(\mathrm{s}_{20, \mathrm{w}}\right)\right]$ distributions for mMLKL 1-158 and mMLKL 1-169. f Gel filtration of truncations, mMLKL 1-160, 162, 164 and 166, and mMLKL 1-169 harbouring

monomer, to an oligomeric, membrane-associated effector of necroptosis [21, 22, 23, 24]. The key trigger in this conversion is RIPK3-mediated phosphorylation of the activation loop in MLKL's C-terminal pseudokinase domain to induce exposure of the $\mathrm{N}$-terminal four-helix bundle (4HB) domain [23], which is presumed to induce necroptotic cell death via its membrane permeabilisation activity $[15,25,26]$. The mechanism by which a phosphorylation event in the C-terminal pseudokinase domain leads to exposure of the killer $\mathrm{N}$-terminal $4 \mathrm{HB}$ domain is currently unclear. Here, we explore the hypothesis that the two interdomain helices, termed the 'brace' helices, are key to communicating a phosphorylation event in the pseudokinase domain to exposure of the $4 \mathrm{HB}$ domain to enable its participation in membrane localisation, permeabilisation and cell death. the E161A-I162A, T165A-L166A, N163A-K164A, or K167A-Q168A alanine mutations. Constructs that behave similarly to mMLKL 1-169 are coloured in shades of blue, while those that behave similarly to mMLKL 1-158 are coloured in shades of red. $\mathbf{g}$ Standardised continuous sedimentation coefficient $\left[\mathrm{c}\left(\mathrm{s}_{20, \mathrm{w}}\right)\right]$ distributions for E161AI162A and T165A-L166A. h Standardised continuous sedimentation coefficient $\left[\mathrm{c}\left(\mathrm{s}_{20, \mathrm{w}}\right)\right]$ distributions for N163A-K164A and K167AQ168A

MLKL oligomerisation is an essential step in necroptosis [23], however there is no consensus on the stoichiometry of the complex. Trimers [22, 23, 24], tetramers [21, 22], hexamers [15], and octamers [27] have been reported, as well as data showing forced dimerisation of MLKL is sufficient to cause auto-activation and cell death [24, 26]. The oligomerisation interface is unknown, and there is also contention as to whether disulphide bonds are required for oligomer formation, or merely a consequence of higherorder assembly [15, 21, 22, 25, 28]. Previous studies have shown that recombinant mouse and human MLKL constructs comprising the $4 \mathrm{HB}$ domain and the two brace 'helices can form trimers [23, 24], whereas the pseudokinase (PsK) domain alone does not [29]. Notably, truncated forms of recombinant human MLKL (residues 2-154 or 1-140) lacking portions of the brace region do not oligomerise 
a

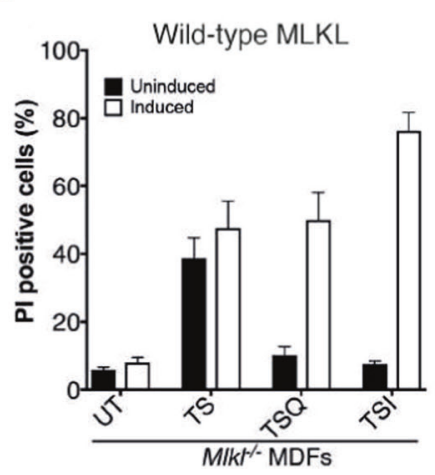

C

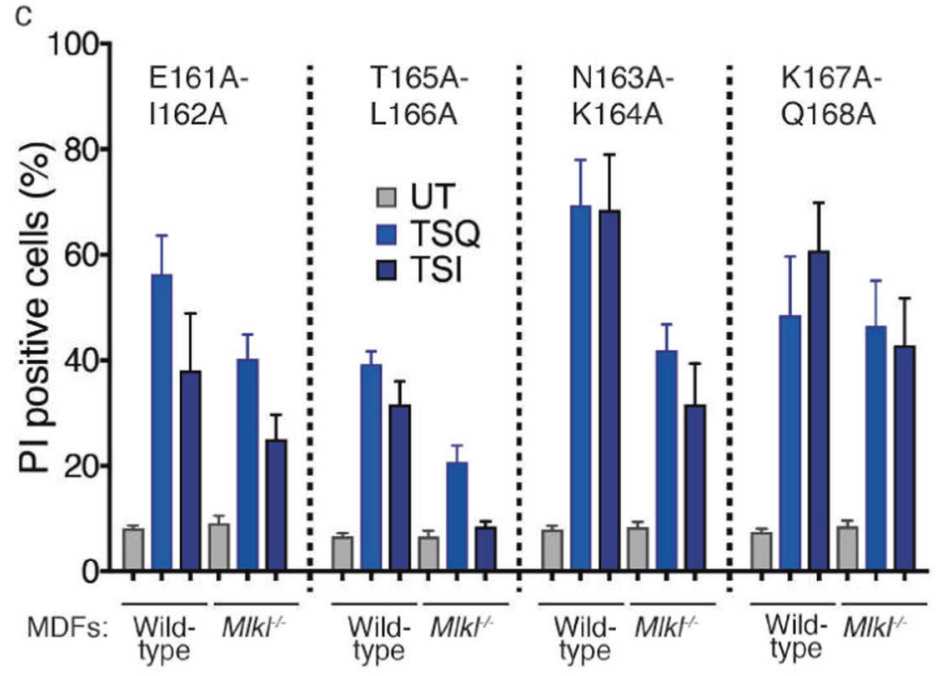

d

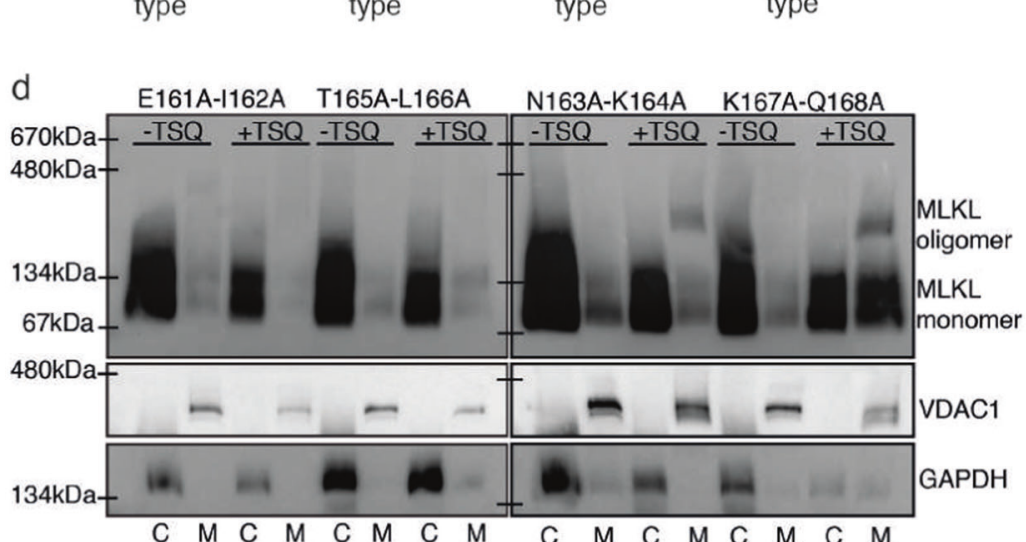

b

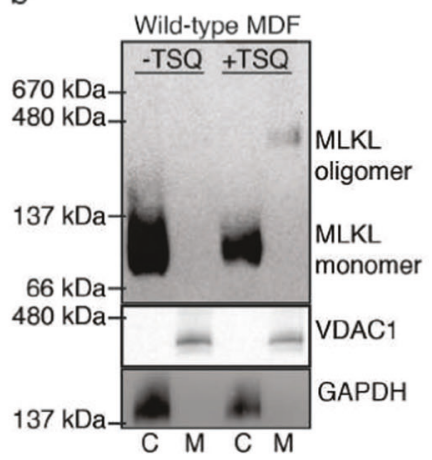

e

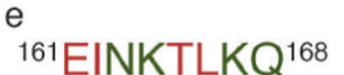

${ }^{161}$ EINKTLKQ ${ }^{16}$
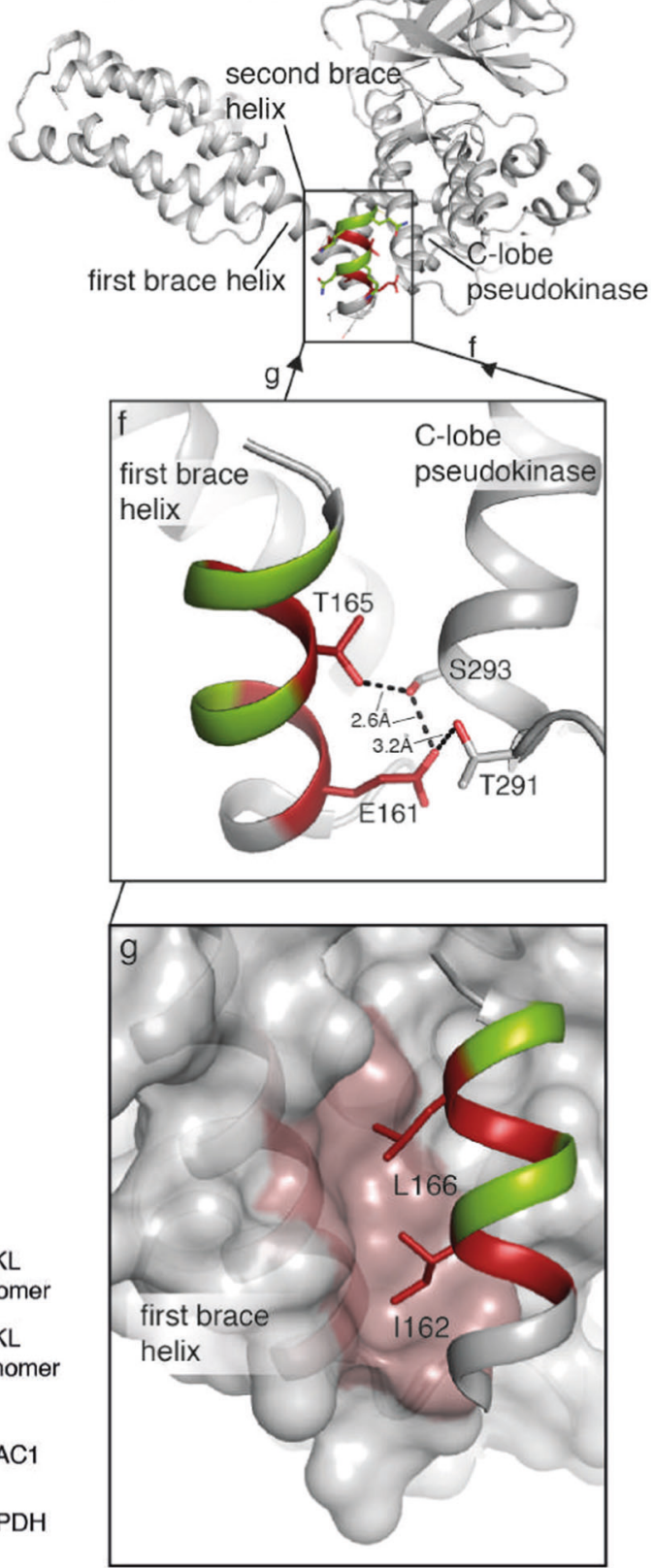

$[24,30]$, implicating the brace region as a possible mediator of oligomerisation. Similar studies are yet to be reported for MLKL orthologues, however, and the precise molecular details remain to be determined.

Involvement of the brace region in the molecular switch mechanism and oligomerisation has so far been inferred. Here, we directly interrogated the function of the MLKL 'brace' helices. In addition to revealing an important role for the second brace helix in mediating oligomerisation and cell death through truncation and mutational analyses, our studies of mouse-human MLKL chimeras-in which the 4HB, brace or pseudokinase domain were swapped between mouse and human MLKL (and vice versa)-indicate that the integrity of the brace helices is crucial to interdomain communication. We observed that disrupting communication between the $\mathrm{N}$ and C-terminal domains of MLKL is capable of flipping the molecular switch to generate constitutively active constructs. A working switch mechanism could be restored by substitution of specific residues in the predicted first brace helix and adjacent loop, such that necroptotic stimuli was required for cell death to ensue. These data highlight the importance of the brace region to 
Fig. 2 Cellular activity of MLKL bearing second brace helix mutations parallels behaviour of recombinant proteins. a $M l k l^{-1}$ MDFs reconstituted with wild-type MLKL were stimulated with TNF (T), Smacmimetic (S) and Q-VD-OPh (Q) or emricasan/IDN-6556 (I), and cell death was quantified using propidium iodide (PI) staining and flow cytometry. b Western blot of Blue-Native PAGE of wild-type MDFs treated \pm TSQ to measure localisation to cytoplasmic (C) or membrane (M) fractions and oligomerisation state. VDAC1 and GAPDH western blot reprobes were used to assess quality of fractionation. $\mathbf{c}$ Full-length MLKL constructs containing indicated alanine mutations were stably incorporated into MDFs via lentiviral infection, and protein expression induced by doxycycline (dox) treatment. Cells were either left untreated (UT), or treated with TSQ or TSI to induce necroptosis $3 \mathrm{~h}$ post dox induction. Cell death was measured using PI staining and flow cytometry $24 \mathrm{~h}$ after death stimulation. Responses to an apoptotic stimulus (TS), and death in the presence or absence of dox-induction, are shown in entirety in Supplementary Figure 2. d Western blot of Blue-Native PAGE on cell fractions was used to monitor the formation of MLKL higher-order species and determine cellular localisation in untreated or TSQ stimulated $M l k l^{--}$MDFs, expressing alanine mutant constructs. C; cytoplasmic fraction, M; membrane fraction. Western blots for VDAC1 (membrane associated) and GAPDH (cytoplasmic protein) were used to assess the quality of fractionation. BN-PAGE blots are representative of 2 independent experiments. Cell death data are the mean \pm s.e.m. of three independent experiments with 2-3 biologically independent replicates per experiment $(n=6-9)$. e Residues subjected to alanine mutagenesis were mapped on to the fulllength mouse MLKL structure (PDB: 4BTF) [1]. Mutation of residues in green (N163, K164 and K167, Q168) does not preclude oligomerisation, while those in red (E161, I162 and T165, L166) block oligomerisation. f Zoomed depiction of second brace helix structure in e. Hydrogen bonds between the second brace helix (E161 and T165) and C-lobe of the pseudokinase domain (T291 and S293) are indicated in black dashed lines with interatomic distances annotated. $\mathbf{g}$ Atoms from the first brace helix that form hydrophobic packing interactions with I162 and L166 from the second brace helix are coloured pink and shown in a surface representation

two related facets of MLKL activation: as an interface that contributes to MLKL oligomerisation, and in the relay of the proposed molecular switch mechanism to connect pseudokinase domain phosphorylation to the release or activation of the $4 \mathrm{HB}$ domain executioner function.

\section{Results}

\section{Residues in the second brace helix mediate trimerisation of mouse MLKL N-terminal domain}

Our previous analytical ultracentrifugation (AUC) analyses showed that the mouse MLKL (mMLKL) 4HB + brace (residues 1-169) exists in solution in vitro in a monomertrimer equilibrium with a $K_{\mathrm{D}}$ for self-association of $7.2 \mu \mathrm{M}^{2}$ (ref. 23). Subsequent biophysical studies of human MLKL (hMLKL) constructs indicated that while a construct incorporating the 4HB and two brace helices (1-182) oligomerised, truncation of one (1-154) or both (1-140) brace helices compromised oligomerisation $[24,30]$. Here we sought to validate whether the brace region of mMLKL mediates oligomerisation, and to perform detailed mutational analyses to more clearly map the intersubunit interface.

Initially, we generated an mMLKL construct encompassing the 4HB domain and first brace helix (residues 1-158), as defined from our earlier crystal structure of mMLKL [1]. Compared to the homotrimeric mMLKL 1-169, which encompasses the $4 \mathrm{HB}+$ two brace helices (Fig. 1a, b), mMLKL 1-158 was monomeric. First, mMLKL 1-158 eluted at a higher retention time from analytical size exclusion chromatography than mMLKL 1-169 (Fig. 1c). Second, by sedimentation velocity analytical ultracentrifugation (SV-AUC), we observed a single peak with sedimentation coefficient of 1.8 for mMLKL 1-158 (Fig. 1d), while two species with sedimentation coefficients of 2.1 and 3.3 were observed for mMLKL 1-169 (Fig. 1e), consistent with its propensity for selfassociation into higher-order species. Sequential deletion of terminal residues in the second brace helix led to behaviour comparable to mMLKL 1-158 in analytical size exclusion chromatography (Fig. 1b,f; Supplementary Figure 1a), indicating that integrity of the second brace helix is crucial to higher-order assembly in solution.

To further define which residues mediate mMLKL trimerisation, we substituted pairs of residues in the second brace helix of mMLKL 1-169 with alanine to generate E161A-I162A， N163A-K164A， T165A-L166A and K167A-Q168A mutants (Fig. 1b, Supplementary Figure 1a). Analytical size exclusion chromatography and SVAUC experiments identified two mutant constructs, N163AK164A and K167A-Q168A, which retained the ability to form trimers (Fig. 1h), and two constructs, E161A-I162A and T165A-L166A, which were primarily monomeric (Fig. 1g). These results indicate that residues E161, I162, T165 and L166, which are involved in the packing of the second brace helix against the first brace helix and the pseudokinase domain in the mMLKL structure, contribute to mMLKL oligomerisation.

\section{Trimerisation via the second brace helix is necessary for necroptosis}

To test whether residues that were important for mMLKL 1-169 oligomerisation, E161, I162, T165, and L166, play an important role in necroptosis, we introduced alanine substitutions into full-length mouse MLKL and examined their capacity to induce death in response to necroptotic stimuli. Doxycycline (Dox)-inducible wild-type or mutant mMLKL lentiviral constructs were stably transduced into wild-type and $\mathrm{Mlkl}^{-/}$mouse dermal fibroblasts (MDFs), and their effects on cell viability measured by propidium iodide (PI) staining using flow cytometry following no stimulation 
(untreated, UT), an apoptotic (TNF, T; Smac mimetic, S), or necroptotic (TS; Q-VD-OPh, Q; or emricasan/IDN-6556, I) stimulus (Fig. 2; Supp. Figure 2). Many experimenters induce necroptosis with the pan-caspase inhibitor, Q-VDOPh (Q) [1, 19, 23, 26, 31], however emricasan (IDN-6556, I) has recently been shown to inhibit caspase- 8 hetero- and homodimers [32] more potently and might therefore be a better tool than Q-VD-OPh. Comparing stimulation of necroptosis with TSQ and TSI side-by-side, revealed TSI to be a more potent driver of necroptosis in $M l k l^{-/}$mouse fibroblasts reconstituted with inducibly expressed wild-type mMLKL (Fig. 2a). Our previous work has established that under basal conditions, mMLKL is cytoplasmic and exists as a presumed monomer [19, 23, 31]. Upon stimulation with necroptotic stimuli, such as TSQ, mMLKL translocates to membranes and assembles into a higher molecular weight species whose assembly can be monitored by Blue-Native (BN) PAGE (Fig. 2b).

The ability of each mutant to reconstitute the necroptosis pathway was assessed upon induction of protein expression in both wild-type and $M l k l^{-/-}$MDFs (Fig. 2c). All cell lines expressed MLKL constructs comparably (Supplementary Figure 3). Full-length mMLKL constructs harbouring the alanine mutations that prevented oligomerisation of mMLKL 1-169 in analytical size exclusion chromatography and AUC, E161A-I162A and T165A-L166A, showed reduced ability to kill in both wild-type and $M l k l^{-/-}$ MDFs (Fig. 2c) compared to wild-type mMLKL (Fig. 2a). The capacity of mMLKL to induce necroptosis was almost entirely abrogated by the T165A-L166A mutation, while E161A-I162A mMLKL exhibited attenuated, but measurable, cell death upon TSQ or TSI stimulation (Fig. 2c). In BN-PAGE experiments, in contrast to wild-type mMLKL (Fig. 2b), neither mutant formed detectable oligomers $6 \mathrm{~h}$ post TSQ treatment (Fig. 2d), consistent with deficits in the capacity of these mutants to induce cell death.

Expression of full-length mMLKL constructs harbouring mutations that were capable of forming oligomers in the context of recombinant mMLKL 1-169, N163A-K164A and K167A-Q168A, in wild-type and $M l k l^{-/}$MDFs led to levels of death similar to that observed of wild-type MLKL upon TSQ stimulation ( $45 \%$ death), although to a lesser extent when treated with TSI (Fig. 2c). Like wild-type mMLKL, both mutants translocated to the membrane fraction and assembled into oligomers in BN-PAGE experiments after $6 \mathrm{~h}$ of treatment with TSQ (Fig. 2d).

In the context of the full-length mouse crystal structure, residues which do not influence oligomerisation (N163, K164, K167 and Q168) are solvent facing (green in Fig. 2e), whereas those that disrupt trimerisation when mutated to alanine face their two flanking regions: the first brace helix and pseudokinase domain (red, Fig. 2e). E161 and T165 form hydrogen bonds with T291 and S293 located on the C-lobe of the pseudokinase (PsK) domain (Fig. 2f), while I162 and L166 interact with a shallow hydrophobic pocket on the first brace helix (Fig. 2g). These data implicate the second brace helix as a key determinant of mMLKL trimerisation and assert the functional importance of mMLKL trimerisation in necroptotic signalling.

\section{The mouse MLKL 4HB + brace adopts a radial trimer conformation in solution}

To gain a structural insight into the topology of the mMLKL N-terminal region in solution, we analysed recombinant mMLKL 1-169) using Small Angle X-ray Scattering (SAXS; Supplementary Table 1). To ensure the mMLKL (1-169) monomer-oligomer equilibrium favoured the oligomer form, protein at $7 \mathrm{mg} / \mathrm{mL}(>340 \mu \mathrm{M})$, a markedly higher concentration than the $K_{\mathrm{D}}$ for trimerisation $\left(7.2 \mu \mathrm{M}^{2}\right)$ previously reported for this protein [23], was resolved by inline size exclusion chromatography eluting protein into the path of the X-ray beam at the Australian Synchrotron SAXS/WAXS beamline. The resulting data were of high quality, with no indications of polydispersity evident from Guinier analysis (Fig. 3a; inset), with the interatomic distance distribution indicating a maximum dimension of $100 \AA$ (Fig. 3b). Rigid body fitting of the mMLKL 1-169 coordinates (from our full-length mMLKL crystal structure [1]) using SASREF with P3 symmetry led to a trimeric model with excellent agreement between experimental data and theoretical scattering calculated for the trimeric model, as signified by $\chi=0.60$. This model is consistent with our mutational analyses: the second brace helix serves an important function as an inter-protomer contact point (Fig. 3c; face of helix with E161, I162, T165, L166 shown in red); and key residues on the $\alpha 4$-helix of the 4HB domain required for MLKL to execute necroptosis identified in our previous work [23] are presented on one face of the trimer model. Furthermore, these data were only consistent with the assembly of the mMLKL 4HB + Brace region into homotrimers, as monomeric and other oligomerisation regimes led to poor fits, with $\chi$ values $>3$.

\section{The MLKL brace region functions as a communication lever between pseudokinase and 4HB domains in a species-specific manner}

Previously, we noted that the species of origin of both MLKL and the host cell line governs the capacity of MLKL to induce cell death. For example, $M l k l^{-/}$MDFs cannot be reconstituted by expression of hMLKL [26]; and mouse, but not human, MLKL 4HB constitutively kill cells in the absence of stimuli [26] (summarised in Fig. 4c). Furthermore, cell death was not induced by expression of fulllength hMLKL, h4HB + Brace and $\mathrm{m} 4 \mathrm{HB}+$ Brace in the 

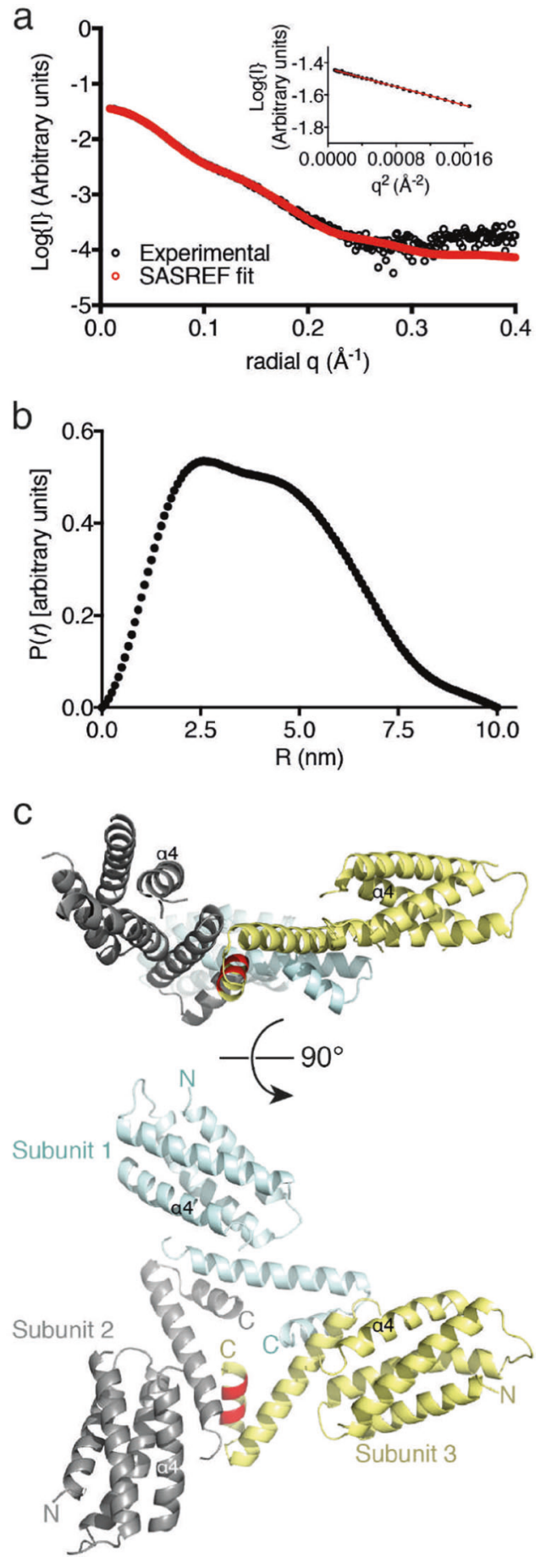

human cell lines, HT29 and U937, in the absence of stimulation of the endogenous necroptotic machinery with TSQ [26] (summarised in Fig. 4c). Interestingly, the h4HB could induce cell death of HT29, but not U937, in the absence of stimuli [26] (Fig. 4c), consistent with differences in endogenous host cell factors contributing to necroptotic death. There is substantial sequence divergence in the brace
Fig. 3 mMLKL 4HB + Brace forms a radial homotrimer in solution. a Small angle scatter profile of averaged, and background subtracted, data from apex of inline size exclusion peak (black circles). Agreement between the experimental and theoretical scatter (red circles) calculated from a rigid body, trimeric model in SASREF is reflected in the $\chi$-value of 0.60. Inset: Guinier plot of experimental SAXS data for $q$. $\mathrm{Rg}<1.3$. Linearity of the plot indicates that polydisperse (aggregated species) do not contribute measurably to the scatter. b Interatomic distance distribution plot, $\mathrm{P}(\mathrm{r})$, profile calculated from scattering data with GNOM. Maximum particle dimension, Dmax, was estimated as $100 \AA$ A. c Orthogonal views of the trimeric model of mMLKL 4HB + brace. The three units are shown in grey, cyan and yellow, and the residues important for trimerisation are shown in red

region of MLKL from multiple species, including a nineresidue insertion in the human brace region compared to the mouse counterpart (Fig. 4a). To define the extent to which variation in the brace helices would impact MLKL activation, oligomerisation and host cell species-specificity, we prepared a series of mouse:human chimeras. The chimeras were fused between mouse and human sequence at key functional domain boundaries selected for mMLKL based on the full-length crystal structure [1]: 4HB, residues 1-124; brace, 125-174; and pseudokinase domain (PsK), 175-464. Corresponding residues were selected for human MLKL by sequence alignment as 1-125 (4HB), 126-185 (brace), and 186-471 (PsK). Initially, five chimeric constructs were generated; $\mathrm{m} 4 \mathrm{HB}^{1-124}-\mathrm{hBrace}^{126-185}$ $\mathrm{hPsK}^{186-471}, \quad \mathrm{~h} 4 \mathrm{HB}^{1-125}-\mathrm{mBrace}^{125-174}-\mathrm{mPsK}^{175-464}$, $\mathrm{m} 4 \mathrm{HB}^{1-124}-\mathrm{mBrace}^{125-174}-\mathrm{hPsK}^{186-471}, \mathrm{~h} 4 \mathrm{HB}^{1-125}$ ' $\mathrm{hBrace}^{126-185}-\mathrm{mPsK}^{175-464}$, and $\mathrm{m} 4 \mathrm{HB}^{1-124}-\mathrm{hBrace}^{126-185}$ $\mathrm{mPsK}^{175-464}$ (Fig. 4b). With these chimeric constructs, we aimed to determine the role of the brace helices in communication between domains through species-specific compatibilities. Chimeras were expressed upon doxinduction in MDFs and two human cell lines, HT29 and U937, and death of untreated (UT), and cells subjected to necroptotic (TSQ) (Fig. 4d-h) or apoptotic (TS) (Supp. Figure 4) stimuli was assessed. Chimeric proteins were expressed to comparable levels, as assessed by western blot (Supp. Figure 5), indicating that folding or expression defects do not contribute to signalling deficits.

Expression of mouse 4HB kills MDFs [23] (Fig. 4c), and it retained this ability when fused to the human brace and pseudokinase (m4HB-hBrace-hPsK) (Fig. 4d). Furthermore, expression of m4HB-hBrace-hPsK also killed the human cell lines, HT29 and U937 (Fig. 4d). This suggests that the brace region and pseudokinase domain from hMLKL cannot restrain the innate ability of the m4HB to kill cells, in contrast to the mouse brace and pseudokinase domain in the context of unstimulated wild-type mMLKL. The m4HBmBrace-hPsK MLKL chimera also constitutively killed MDFs and, to a lesser extent, human cell lines (Fig. 4e), and m4HB-hBrace-mPsK, showed strong constitutive killing of MDFs (Fig. 4h). These data illustrate that mouse 
a

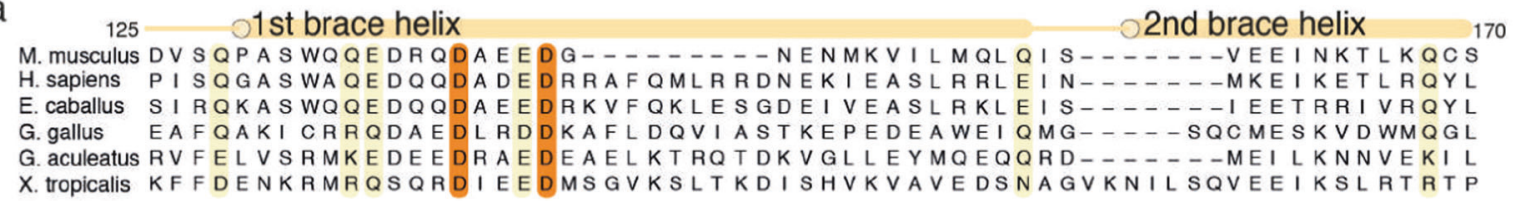

b Human MLKL

$4 \mathrm{HB}$

Brace region

$\mathrm{m}_{4} \mathrm{HB}^{1-124} \mathrm{hBrace}^{126-185} \mathrm{hPsK}^{186-471}$

$\frac{\mathrm{m} 4 \mathrm{HB}^{1-124} \mathrm{mBrace}}{\mathrm{e})}$

$\mathrm{h} 4 \mathrm{HB}^{1-125} \mathrm{mBrace}{ }^{125-174} \mathrm{mPsK} \mathrm{K}^{175-464}$

$(\mathrm{f}, \mathrm{i})$

$\mathrm{h} 4 \mathrm{HB}^{1-125} \mathrm{hBrace}^{126-185} \mathrm{mPsK} \mathrm{g}^{175-464}$

(h) $\mathrm{HHB}^{1-124} \mathrm{hBrace}^{126-185} \mathrm{mPsK} \mathrm{K}^{175-464} \mathrm{OSO}$

d

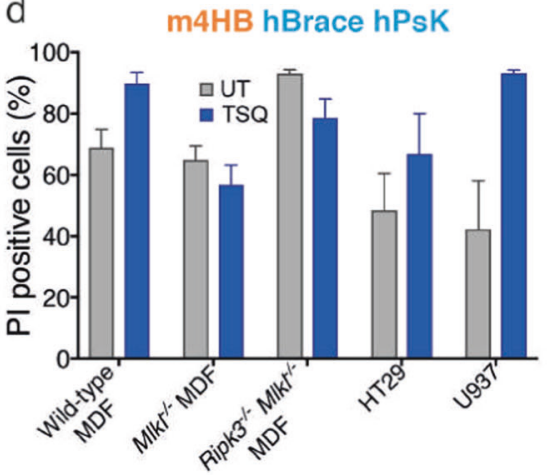

g

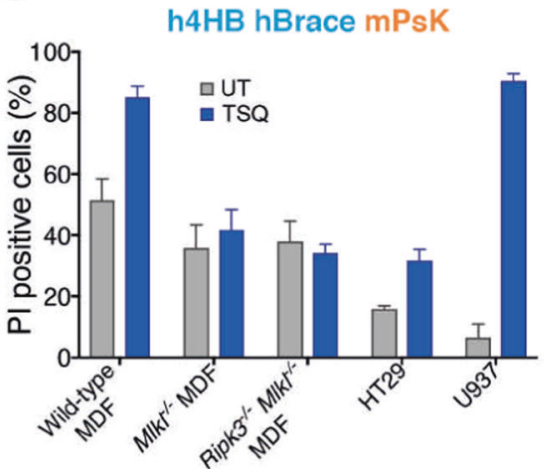

C

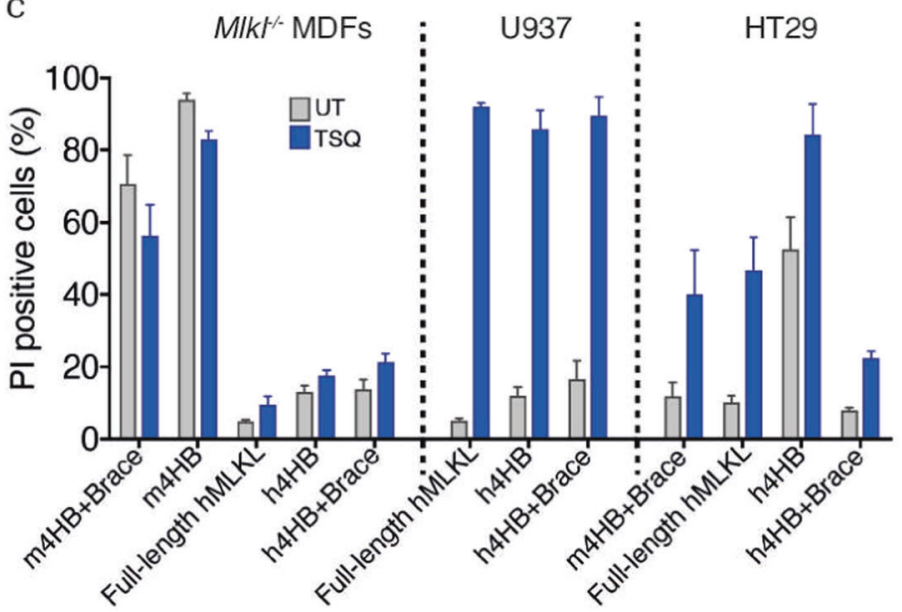

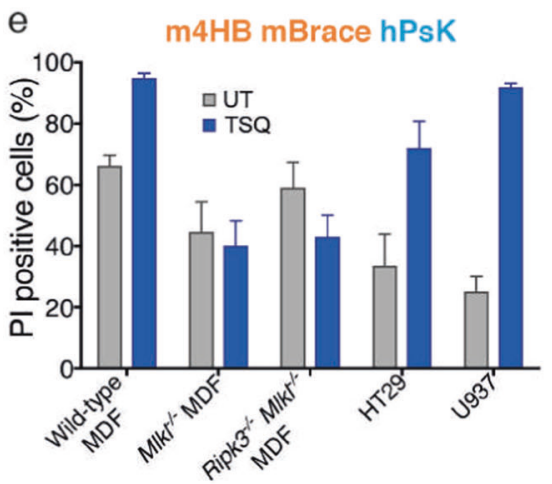

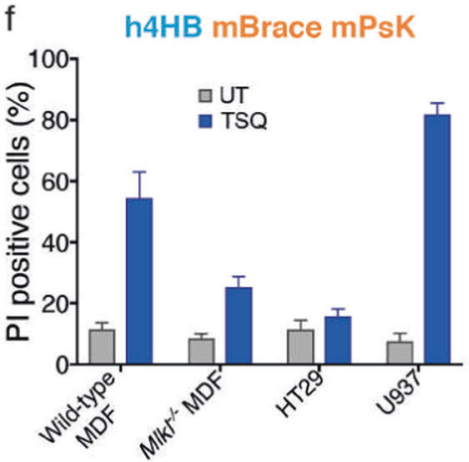

$\mathrm{h}$

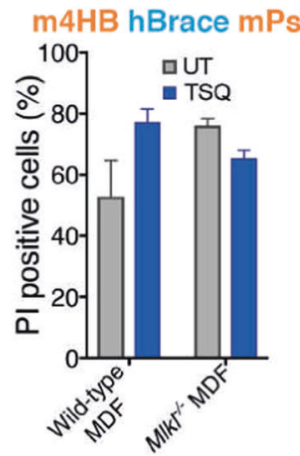

i h4HB mBrace h4HB hBrace

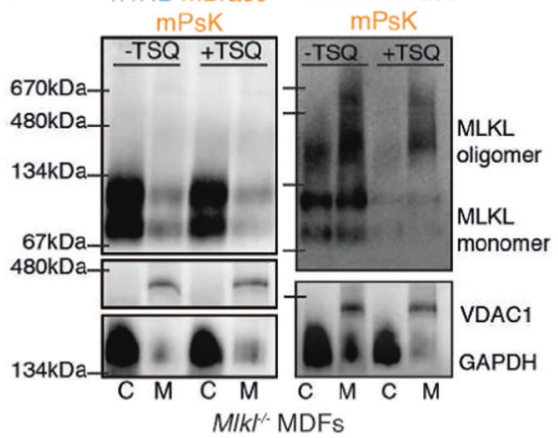

pseudokinase and the mouse brace are required to work together to suppress the m4HB.

To further define species-specificity, we made reciprocal constructs comprising the N-terminus of hMLKL and the
C-terminus of mMLKL. In contrast to m4HB-hBrace-hPsK, but consistent with our published observations on the h4HB domain [26] (Fig. 4c), h4HB-mBrace-mPsK, did not constitutively kill when expressed in either human or mouse 
Fig. 4 Chimeric mouse:human MLKL constructs reveal key determinants for constitutive killing of human and mouse cell lines. a Sequence alignment of MLKL brace regions from multiple species. Residue number for mouse and a schematic of the position of brace helices in the mouse structure are presented above the alignment. b Schematics of mouse (orange) and human (blue) MLKL chimeras. c Mouse $\mathrm{m} 4 \mathrm{HB}+$ Brace (residues 1-180), mouse 4HB (1-125), and (undimerised) gyrase fusions of full-length hMLKL (1-471), h4HB + Brace (1-180) and h4HB (1-125) were inducibly expressed in $M l \mathrm{k}^{-/}$ MDF, human HT29 and U937 cells, and death of untreated or TSQstimulated cells measured by PI-staining and flow cytometry. These data were reported previously [23, 26], and are included for comparison with the chimera and mutant data reported herein. d-h Wild-type, $\mathrm{Mlkl}^{-/}$and $\mathrm{Mlkl}^{--} \mathrm{Ripk3}^{-/-}$MDFs, HT29, and U937 cells were stably transfected with doxycycline inducible chimeric constructs. Following induction of protein expression with dox, cells were either left untreated (UT) or stimulated with TSQ/TSI. MDF cell death was quantified by PI uptake after $24 \mathrm{~h}$; data are the mean \pm s.e.m. of three independent experiments with 2-3 biologically independent replicates per experiment $(n=6-9)$. Death assays on the human cell lines, HT29 and U937, were performed similarly to MDFs, except that cell death was measured at $48 \mathrm{~h}$ post-stimulation. Human cell line data are the mean \pm s.e.m. of three independent experiments $(n=3)$, except for those in $\mathbf{g}$, where $n=2$. Responses to an apoptotic stimulus (TS), and death in the presence or absence of dox-induction, are shown in entirety in Supplementary Figure 4. (i) Western blot of Blue-Native PAGE on fractionated $M l l^{-/}$MDFs expressing inactive and constitutively-activated chimeras treated \pm TSQ. h4HB-hBrace-mPsK cells were harvested $18 \mathrm{~h}$ after dox treatment; h4HB-mBrace-mPsK were harvested at $6 \mathrm{~h}$. $\mathrm{C}$ cytoplasmic fraction, $\mathrm{M}$ membrane fraction. VDAC1 (membrane associated) and GAPDH (cytoplasmic protein) blots were used as fractionation controls. All BN-PAGE blots are representative of two independent experiments

cells, and this construct did not reconstitute necroptotic signalling in $\mathrm{Mlkl}^{-/}$MDFs (Fig. 4f). Furthermore, higherorder oligomers were not observed in $\mathrm{Mlkl}^{-/}$MDFs expressing h4HB-mBrace-mPsK following TSQ stimulation and MLKL remained localised to the cytoplasm in BNPAGE analysis (Fig. 4i), indicating that this chimera is unable to oligomerise and translocate to the plasma membrane. In addition to the MLKL monomer, we detected a second band that migrated near the $134 \mathrm{kDa}$ marker on $\mathrm{BN}$ PAGE, possibly representing a dimer (Figs. $4 \mathrm{i}, 5 \mathrm{~d}$ ). This is distinct from the higher-order species associated with cell death that migrates just below the $480 \mathrm{kDa}$ marker on $\mathrm{BN}$ PAGE. In contrast to the h4HB-mBrace-mPsK chimera, inclusion of the hMLKL brace region within the h4HBhBrace-mPsK construct conferred the ability to constitutively induce cell death when expressed in wild-type, $\mathrm{Mlkl}^{--}$and $\mathrm{Mlkl}^{--}$Ripk3 $^{---}$MDFs (Fig. 4g). Induction of this construct for $18 \mathrm{~h}$ in $\mathrm{Mlkl}^{--}$MDFs led to formation of oligomers by BN-PAGE in the absence of stimuli (Fig. 4i). The same construct did not induce constitutive cell death in the human lines, U937 and HT29 (Fig. 4g). These data implicate the pseudokinase domain as a key adaptor domain that dictates species-selectivity of MLKL and whether an MLKL orthologue can interact with the host machinery.

\section{In concert with the PsK domain, the brace helices act as a latch to restrain the executioner function of the MLKL 4HB domain}

The h4HB-hBrace-mPsK construct constitutively killed MDFs in the absence of stimulus (Fig. 4g), yet only differs from the inactive h4HB-mBrace-mPsK (Fig. 4f) in the brace region. Considering that a necroptotic stimulus (TSQ) does not relieve the repressive interaction within the h4HBmBrace-mPsK chimera or induce oligomerisation, our data favour the existence of an inhibitory interaction between the mouse brace region and the human $4 \mathrm{HB}$ that blocks oligomerisation and therefore MLKL-driven cell death.

To pinpoint the differences that lead to such different killing abilities in MDFs, we made pairwise mutations along the brace helices in the h4HB-hBrace-mPsK construct, substituting human brace residues with counterparts from the mouse sequence (Fig. 5a). These constructs were inducibly expressed in wild-type and $\mathrm{Mlkl}^{-/}$MDFs (Supp. Figure 7), and their capacity to induce cell death in the absence of necroptotic stimuli, or to reconstitute necroptosis upon TSQ or TSI stimulation, were evaluated. Chimeras containing the human to mouse substitutions, $\mathrm{MK}^{\mathrm{h}}>\mathrm{VE}^{\mathrm{m}}$ and $\mathrm{KE}^{\mathrm{h}}>\mathrm{NK}^{\mathrm{m}}$, that lie in the second helix retained the capacity to induce stimulus-independent death of MDFs (Fig. 5b). This suggests that the second brace helix, whilst being important for oligomerisation, does not play a substantive role in tipping the balance between activated and inactive MLKL. The insensitivity of these residues from the second brace helix to mutation is not altogether surprising, given that the in the mouse full-length structure, residues at equivalent points in the helix are all solvent facing.

In contrast, four constructs, harbouring $\mathrm{KI}^{\mathrm{h}}>\mathrm{NM}^{\mathrm{m}}$, $\mathrm{EAS}^{\mathrm{h}}>\mathrm{KVI}^{\mathrm{m}}, \mathrm{RR}^{\mathrm{h}}>\mathrm{MQ}^{\mathrm{m}}$ or $\mathrm{EIN}^{\mathrm{h}}>\mathrm{QIS}^{\mathrm{m}}$ substitutions, no longer exhibited constitutive killing of MDFs, but were able to reconstitute the necroptosis pathway in $\mathrm{Mlkl}^{-1-}$ MDFs, where cell death (Fig. 5c) and membrane translocation/oligomerisation (Fig. 5d) ensued only following treatment with necroptotic stimuli. Despite comprising the human MLKL 4HB + brace and the mouse pseudokinase domain, it was possible to confer the properties of wild-type mMLKL on the construct through subtle changes in the predicted first brace helix and subsequent loop. Substitution of hBrace residues that preclude an inhibitory interaction with the mouse PsK domain within h4HB-hBrace-mPsK enabled restoration of the switch mechanism (summarised in Fig. 6).

\section{Discussion}

The precise molecular details underlying MLKL's activation and cell killing mechanism remain incompletely 
a

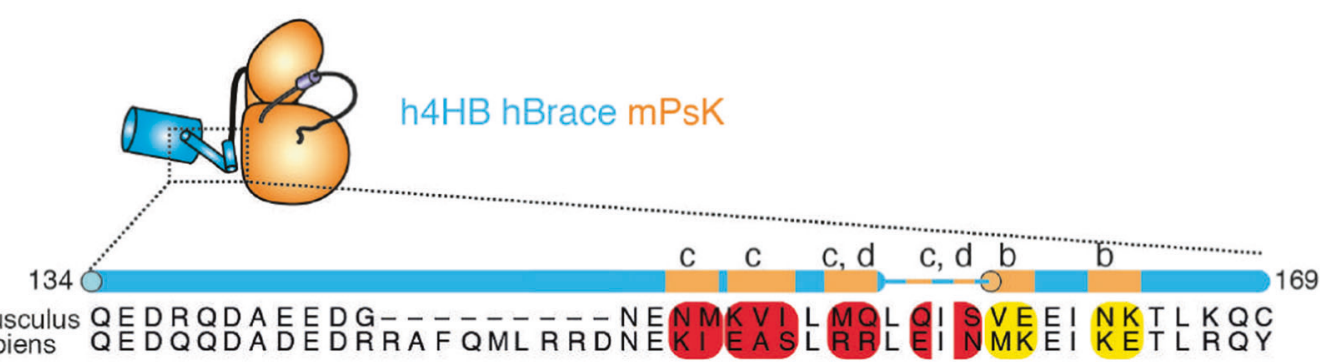

b

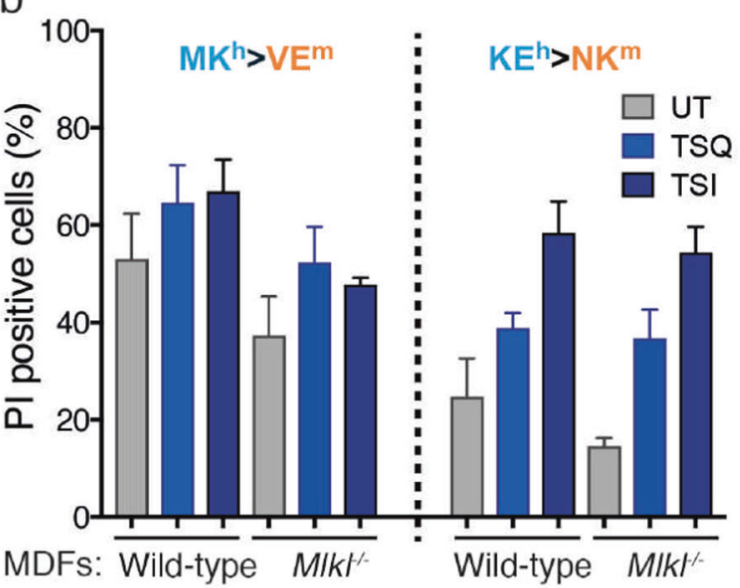

d

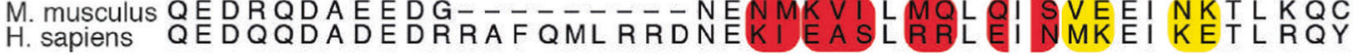

\section{9}
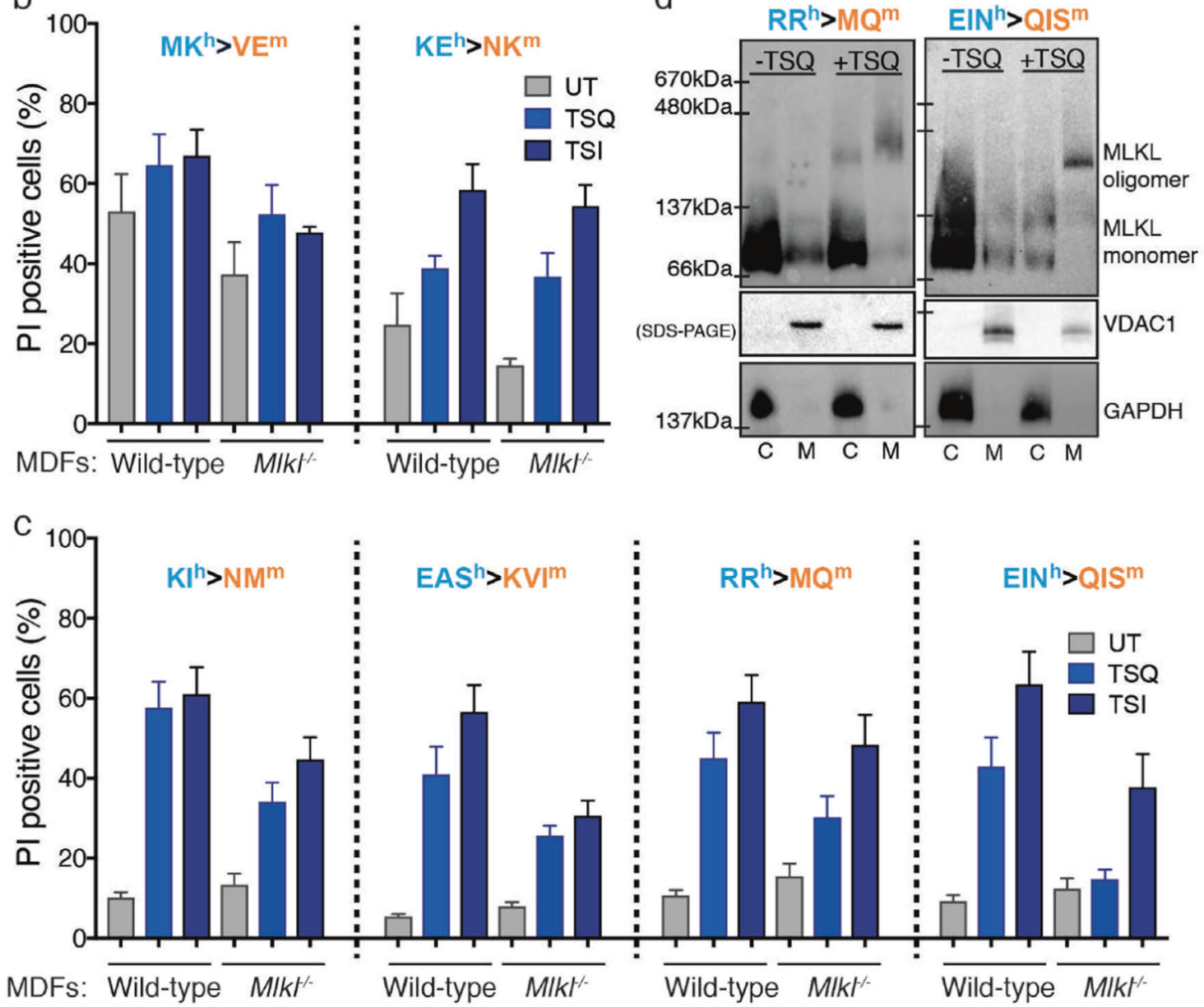

Fig. 5 Residue swaps between human and mouse brace region suggest distinct roles for the first and second brace helix. a Schematic showing the base construct, h4HB-hBrace-mPsK, and the sequence of both mouse and human MLKL at the brace region. Residues mutated from the human sequence to the equivalent mouse residues are highlighted in red (first brace helix and proceeding loop) or yellow (second brace helix). Location of brace helices (based on mMLKL structure [1]), and letters corresponding to figure panels are indicated. b, $\mathbf{c}$ Expression of chimeric constructs harbouring the indicated mutations in wild-type and $\mathrm{Mlkl}^{-/}$MDFs was induced by dox, and cell death in untreated or

necroptotic (TSQ/TSI) conditions measured by PI uptake and flow cytometry. Death assays represent mean \pm s.e.m. of three independent experiments with 2-3 biologically independent replicates per experiment $(n=6-9)$. Responses to an apoptotic stimulus (TS), and death in the presence or absence of dox-induction, are shown in entirety in Supplementary Figure 6. d Western blots of BN-PAGE performed on fractionated cell lysates from h4HB-hBrace EIN $^{\mathrm{h}}>\mathrm{QIS}^{\mathrm{m}} \mathrm{mPsK}$ and h4HB-hBrace RR > MQ mPsK, with and without TSQ stimulation. Blots are representative of two independent experiments

understood. The consensus among published studies is that MLKL activity relies on higher-order assemblies [21, 22, $23,26,28]$, however, the stoichiometry, and the importance of intersubunit disulphide crosslinks and post-translational modifications [33] within the MLKL oligomer remain unclear. Additionally, there have been conflicting reports regarding the capacity of exogenously expressed MLKL 4HB domains to kill cells. Noting that sequences of 


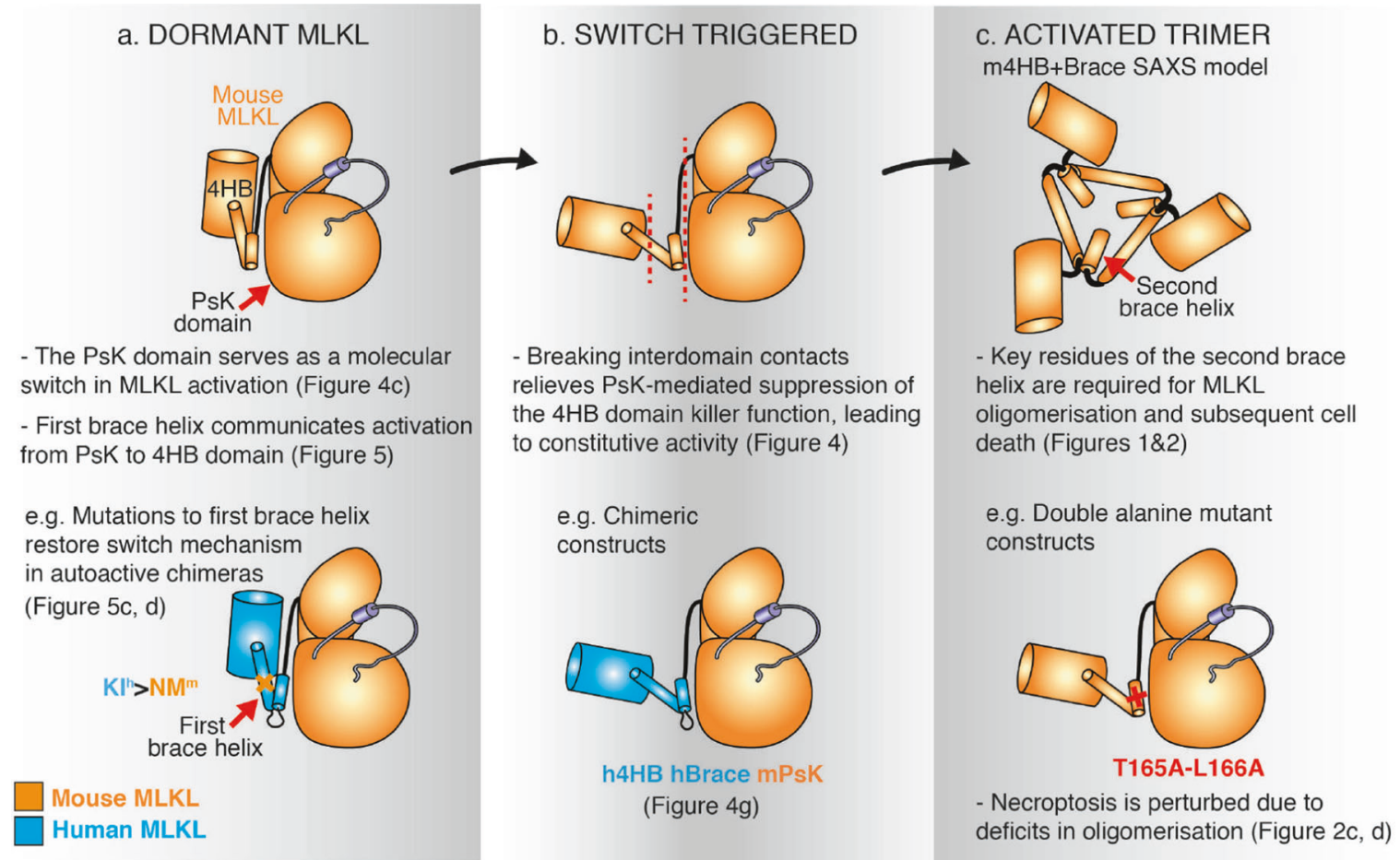

Fig. 6 Summary of the roles of the brace helices in orchestrating the transition of MLKL from dormant to killer oligomeric form. a The pseudokinase domain suppresses the executioner activity of the $4 \mathrm{HB}$ domain in the dormant form of MLKL. Introduction of the $\mathrm{KI}^{\mathrm{h}}>$ $\mathrm{NM}^{\mathrm{m}}, \mathrm{EAS}^{\mathrm{h}}>\mathrm{KVI}^{\mathrm{m}}, \mathrm{RR}^{\mathrm{h}}>\mathrm{MQ}^{\mathrm{m}}$ or $\mathrm{EIN}^{\mathrm{h}}>$ QIS $^{\mathrm{m}}$ mutations (Fig. $5 \mathrm{c}$, d) into the first brace helix and adjacent loop of the constitutively active h4HB-hBrace-mPsK chimera abrogated constitutive activity, and restored responsiveness to necroptotic stimuli. The data implicated the first brace helix and adjacent loop in maintenance of the dormant MLKL species and communication of PsK domain activation to the

the brace helices vary in length and composition among MLKL orthologues, we sought to establish the extent to which the brace helices govern MLKL activation via oligomerisation and communication between the PsK and 4HB domains.

Previously, we reported that mMLKL $4 \mathrm{HB}+$ brace (residues 1-169) assembled into homotrimers in vitro [23]. Subsequently, an NMR study of hMLKL 4HB + first brace helix (2-154) [30] and a SAXS study of the hMLKL PsK [29] demonstrated that both of these domains exist as stable monomers. From these data, we predicted that the second brace helix was a key determinant in MLKL oligomerisation. From our current analyses, we deduced that any truncations to the second brace helix of mMLKL (residues 159-169) compromise homotrimer assembly, with alanine scanning mutagenesis pinpointing E161/I162 and T165/ L166 as key determinants. Full-length mMLKL with these mutations failed to assemble into higher molecular weight complexes and were compromised in their ability to execute necroptosis. The T165A/L166A mutation almost completely abrograted mMLKL-mediated death, while the
4HB domain. b Four of the five chimeric constructs generated exhibited constitutive killing activity, highlighting that the native interface between each subdomain (the $4 \mathrm{HB}$, the brace and the PsK) conferred by either mouse or human sequence, is required for maintenance of the molecular switch. Breaking these interfaces triggers the switch for MLKL, resulting in a species primed to kill (Fig. 4). c Key residues of the second brace helix mediate oligomerisation of the mouse 4HB + Brace (Fig. 1), which forms trimers in solution (Fig. 3). These residues are required for oligomerisation of full-length MLKL and consequent necroptosis in MDF cells (Fig. 2)

E161A/I162A mMLKL retained the capacity to induce necroptotic cell death after $24 \mathrm{~h}$. This incomplete abrogation of cell death suggests that the full-length MLKL E161AI162A mutant can still oligomerise, albeit with delayed kinetics, as no oligomer is observed after $6 \mathrm{~h}$ in Blue-Native PAGE. This in turn suggests that in the context of the fulllength protein, there are additional interfaces/interactions apart from those targeted by the alanine substitutions, that stabilise the trimer.

To further dissect the role of the brace helices in MLKL activation, and whether the brace helices contribute to the species-specificity previously noted in MLKL-mediated death of human and mouse cell lines [26], we generated chimeric constructs containing combinations of mouse and human MLKL subdomains. These studies implicated the host species brace + PsK as serving a modulatory role in restraining or promoting the $4 \mathrm{HB}$ domain-mediated cell death, depending on the cellular context. In mouse cells, we showed that it is the species-specific mBrace and mPsK together that are essential for repressing the $\mathrm{m} 4 \mathrm{HB}$, as any chimera containing the $\mathrm{m} 4 \mathrm{HB}$ caused constitutive cell 
death. On the other hand, the h4HB-hBrace-mPsK construct, which caused constitutive cell death in MDFs independent of mRIPK3, did not cause constitutive death in HT29 or U937 cells, implicating the hPsK as an important determinant of MLKL-driven necroptosis in human cells. Intriguingly, whilst the h4HB-hBrace-mPsK chimera exhibited stimulus-independent killing of mouse fibroblasts, the h4HB fused to the mouse brace + PsK was completely inactive. Based on this finding, we exploited the contrasting killing activities to better understand determinants that suppress the $4 \mathrm{HB}$ domain executioner function. Mutational analyses revealed residues at the end of the predicted first brace helix and in the subsequent loop as key elements of the molecular switch mechanism, which communicates activating phosphorylation events in the PsK domain to the executioner 4HB domain. Our data are most consistent with a role for the first brace helix mutants disfavouring spontaneous assembly of MLKL oligomers, or enabling inhibitory interactions with the pseudokinase domain to be restored. Furthermore, our chimera data reveal an underappreciated role of the MLKL pseudokinase domain in conferring host specificity. h4HB or h4HB + brace constructs do not induce death of mouse fibroblasts [26], and full-length human MLKL does not reconstitute necroptotic signalling in $M l k l^{-/}$fibroblasts [26, 34], even with forced dimerisation [26]. Here we observed that fusion to the mouse PsK domain was sufficient to enable the h4HB + brace, but not the h4HB alone, to kill MDFs. These findings indicate that the mMLKL PsK domain may function as a protein interaction domain to recruit host factors that contribute to necroptosis signalling, akin to the role reported for the co-chaperone system, HSP90:Cdc37, in MLKL activation $[31,35,36]$. The h4HB-hBrace-mPsK construct constitutively killed Ripk $^{-/-} \mathrm{Mlkl}^{-/-}$fibroblasts, therefore the constitutive oligomerisation observed by BN-PAGE, and killing activity of this construct cannot be explained by constitutive activation by RIPK3.

In the present work, we defined a crucial role for the second brace helix of mouse MLKL in facilitating trimer formation and necroptotic cell death (Fig. 6c), and the first brace helix and adjacent interhelix loop as important regulatory elements that convey the activation signal from the pseudokinase domain to the executioner 4HB domain (Fig. 6a). Collectively, these data illustrate that the brace helices are important regulatory elements in MLKL activation, and not simply an interdomain linker, whose regulatory functions appear to have co-evolved with its neighbouring domains and diverged among species. Owing to their central role in MLKL activation, future studies should consider the contribution of the brace helices to the molecular switch mechanism and in efforts to pharmacologically target MLKL to modulate necroptotic activity in human pathologies.

\section{Materials and methods}

\section{Expression constructs}

Insert DNA was amplified from a mouse MLKL template (synthesised by DNA2.0, CA) by PCR using manuallydesigned oligonucleotides (Integrated DNA Technologies). For recombinant protein constructs, DNA inserts were cloned into vector $\left(\mathrm{Kan}^{\mathrm{r}}\right)$ pETNusH HTB (derived from pETM60) [37, 38], using BamHI and EcoRI restriction sites as an inframe fusion C-terminal to a TEV-protease cleavable NusA-His 6 tag. The mouse MLKL (1-169) construct is as described previously [23], and harbours a C169S substitution. Inserts were Sanger sequenced (AGRF, Parkville, VIC; or Micromon, Clayton, VIC). For mammalian cells, mutations were encoded by oligonucleotides (IDT) and introduced into full-length mMLKL or h4HB-hBracemPsK templates by overlap PCR with terminal BamHI and EcoRI restriction sites used to clone into the $\left(\mathrm{Amp}^{\mathrm{r}}\right)$ pFTRE3G PGK puro vector [1]. Chimeric DNA inserts were synthesised by Bioneer (South Korea) and subcloned analogously into pFTRE3G PGK puro. HEK239T cells at $80 \%$ confluency in $10 \mathrm{~cm}$ plates were transfected with $1 \mu \mathrm{g}$ of pFTRE3G plasmid, helper plasmids pCMV- $\delta$ R8.2 (2.0 $\mu \mathrm{g})$ and pVSV-G $(0.8 \mu \mathrm{g})$ to produce the lentiviral capsid and envelope, and a pGFP $(0.1 \mu \mathrm{g})$ reporter plasmid. After $48 \mathrm{~h}$, the virus containing media was filtered and polybrene (10 $\mu \mathrm{g} / \mathrm{ml}$; Sigma) added. The virus was used to infect six biologically independent Mouse Dermal Fibroblast (MDF) cell lines (three wild-type and three $M l k l^{-/}$, derived from different mice), at $60 \%$ confluency in a six-well plate. After $48 \mathrm{~h}$ puromycin $(5 \mu \mathrm{g} / \mathrm{mL})$ media was added to select cells harbouring the pFTRE3G plasmid.

\section{Recombinant protein expression and purification}

mMLKL NTD constructs were expressed in BL21CodonPlus cells. One liter Super broth cultures containing $50 \mu \mathrm{g} / \mathrm{mL}$ kanamycin were incubated at $37{ }^{\circ} \mathrm{C}$ until optical density at $600 \mathrm{~nm}$ reached $0.6-0.8$, cultures were transferred to an $18^{\circ} \mathrm{C}$ incubator for $15-20 \mathrm{~min}$ before adding $500 \mathrm{mM}$ IPTG (Gold Biotechnology) to induce protein expression. Cultures were incubated overnight $\left(18^{\circ} \mathrm{C}\right)$ and cells harvested by centrifugation $(10,000 \times g, 10 \mathrm{~min})$.

Proteins were expressed with a Tobacco Etch Virus (TEV) cleavable hexa-histidine tagged NusA tag fused to their N-terminus. Cell pellets were resuspended in lysis buffer $(20 \mathrm{mM}$ Tris-HCL, pH $8.0,150 \mathrm{mM} \mathrm{NaCl}, 10 \mathrm{mM}$ imidazole, $\mathrm{pH} 8.0,2 \mathrm{mM} \quad \beta \mathrm{ME}$ ) with $3 \mathrm{mM} \mathrm{MnCl}_{2}$ and DNase (10 U; Roche). Cells were lysed with an EmulsiflexC5 homogeniser (Avestin). Immediately postlysis $1 \mathrm{mM}$ of protease inhibitor phenylmethanesulfonyl fluoride (PMSF; Sigma) was added. The whole lysate was 
centrifuged $\left(45,000 \times g, 20 \mathrm{~min}, 4^{\circ} \mathrm{C}\right)$, and the supernatant $0.2 \mu \mathrm{M}$ filtered. The supernatant was passed over a $\mathrm{Ni}^{2+}$ charged $1 \mathrm{~mL}$ HiTrap Chelating HP cartridge column (GE Healthcare) twice. The column was washed extensively with lysis buffer supplemented with $350 \mathrm{mM} \mathrm{NaCl}$, followed by lysis buffer. Protein was eluted in lysis buffer with $100 \mathrm{mM}$ imidazole. The eluate was buffer exchanged to reduce imidazole concentration, and concentrated, then cleaved overnight $\left(4{ }^{\circ} \mathrm{C}\right)$ with $0.5 \mathrm{mg}$ of hexa-histidine tagged TEV protease. The next day, eluate was passed over a nickel cartridge to remove TEV protease, any uncleaved material and cleaved NusA. The flowthrough containing the NTD construct was loaded onto a HiLoad 16/600 Superdex75 PG size exclusion column (GE Healthcare) and 1.5 $\mathrm{mL}$ fractions collected. Protein purity was assessed by SDSPAGE with Coomassie Brilliant Blue staining. Pure fractions were pooled, concentrated, and TCEP (Thermofisher) added to $1 \mathrm{mM}$.

\section{Qualitative size exclusion chromatography}

All NTD constructs were run on the same Superdex75 10/ $300 \mathrm{GL}$ column (GE Healthcare), at $4{ }^{\circ} \mathrm{C}$, in TBS $(20 \mathrm{mM}$ Tris $\mathrm{pH} 7.5,150 \mathrm{mM} \mathrm{NaCl}$ ), on the same day. Three hundred microliter at $1 \mathrm{mg} / \mathrm{mL}$ was loaded for each protein, and $0.5 \mathrm{~mL}$ fractions collected.

\section{Analytical ultracentrifugation}

Sedimentation velocity experiments were performed using an XL-I analytical ultracentrifuge (Beckman Coulter) equipped with UV/Vis scanning optics. Buffer reference (20 mM Tris-HCl, $150 \mathrm{mM} \mathrm{NaCl}, \mathrm{pH} 8.0,1 \mathrm{mM}$ TCEP) and $380 \mu \mathrm{L}$ of mMLKL sample solutions at $40 \mu \mathrm{M}$ were loaded into $12 \mathrm{~mm}$ double-sector cells with quartz windows and the cells were mounted in an An-60Ti 4-hole rotor or An-50Ti 8-hole rotor. All experiments were conducted at 50,000 r.p.m. $(201,600 \times g)$ and $20^{\circ} \mathrm{C}$, and radial absorbance data were collected at $280 \mathrm{~nm}$ in continuous mode. Data were fitted to a continuous sedimentation coefficient distribution [c(s)] model and converted to standardised sedimentation coefficient $\left(\mathrm{s}_{20, \mathrm{w}}\right)$ distributions using SEDFIT [39]. The protein partial specific volumes, buffer density, and buffer viscosity were calculated using SEDNTERP [40].

\section{Small angle X-ray scattering (SAXS)}

SAXS data were collected on the SAXS/WAXS beamline (Australian Synchrotron) using an inline size-exclusion chromatography setup [41]. Two-dimensional scattering data were radially averaged, normalised to sample transmission, and $11 \times 2 \mathrm{~s}$ scatter patterns from the apex of the elution peak were averaged before background scatter (estimated from the average of 20 scatter patterns from the SEC run prior to protein elution) was subtracted using the ScatterBrain software (written by Stephen Mudie, Australian Synchrotron). The ATSAS suite of software was used for all subsequent SAXS data analyses: Guinier analysis was performed using PRIMUS [42]; the pairwise intraatomic distance distribution function $P(\mathrm{r})$ and $D_{\max }$, the maximum dimension of the particle, were calculated from scattering data using GNOM [43]; rigid body modelling of the mMLKL(1-169) homotrimer was performed using SASREF with P3 symmetry [44]. All structural models were illustrated using PyMOL. The data collection and processing statistics are presented in Supplementary Table 1.

\section{Cell lines}

MDFs were cultured in Dulbecco's Modified Eagle Medium (Life Technologies) supplemented with 10\% (v/v) Foetal Calf Serum, penicillin $(100 \mathrm{U} / \mathrm{mL})$, streptomycin $(100 \mu \mathrm{g} /$ $\mathrm{mL})$ and, after selection, puromycin $(5 \mu \mathrm{g} / \mathrm{mL})$. U937 and HT29 cells were cultured in human tonicity RPMI medium supplemented with $8-10 \% \mathrm{v} / \mathrm{v}$ fetal calf serum (FCS) and puromycin $(5 \mu \mathrm{g} / \mathrm{mL})$ for lines stably transduced with MLKL expression constructs.

\section{Death assays}

MDFs were seeded at $5 \times 10^{4}$ cells/well into 24 -well plates, and allowed to adhere overnight. Cells were treated with 10 $\mathrm{ng} / \mathrm{ml}$ doxycycline, and after $3 \mathrm{~h}$ either left untreated, or treated with TNF (Produced in-house; $100 \mathrm{ng} / \mathrm{mL}$ ) and Smac-mimetic (Compound A, Tetralogic; $500 \mathrm{nM}$ ) (TS), or TS and IDN-6556 (Idun Pharmaceuticals) $(5 \mu \mathrm{M})$ (TSI) or Q-VD-OPh $(10 \mu \mathrm{M})$ (TSQ). Doxycycline negative controls were also performed. $24 \mathrm{~h}$ after treatment, cells were harvested and treated with propidium iodide (PI; $100 \mathrm{ng} / \mathrm{mL}$ ), and PI positive cells counted by BD FACSCalibur flow cytometry. Human cell death assays were conducted analogously, except doxycycline treatment was for $4 \mathrm{~h}$. Cell viability was quantitated by PI staining and flow cytometry $48 \mathrm{~h}$ post-stimulation for human cell lines.

\section{Blue-Native PAGE}

MDFs were seeded at $5 \times 10^{5}$ cells/well in six-well plates, and allowed to adhere overnight. Cells were treated with 10 $\mathrm{ng} / \mathrm{ml}$ dox for $3 \mathrm{~h}$, before being treated with TSQ or left untreated. After $\sim 6 \mathrm{~h}$, when cell death had reached $\sim 40 \%$ by eye, cells were harvested. Membrane fractionation and Blue-Native PAGE (BN-PAGE) was performed as previously described [23]. Briefly the cells were permeabilised 
in MELB buffer with $0.025 \%$ digitonin, and the cytosolic and crude membrane fraction separated by centrifugation. The crude membrane fraction was further solubilised in $1 \%$ digitonin and centrifuged to clarify. The cytosolic fraction was made up to $1 \%$ digitonin, and samples separated on a 4-16\% Bis-Tris Native PAGE gel (Life Technologies). The gel was transferred onto a PVDF membrane (Osmonics), de-stained and denatured before being probed by western blot.

\section{Western blot}

Following transfer via electrophoresis, PVDF membranes were rinsed in PBS-T and blocked with $5 \% \mathrm{w} / \mathrm{v}$ milk powder in PBS-T for $1 \mathrm{~h}$ (RT). They were washed for $3 \times$ 10 min in PBS-T, then incubated overnight $\left(4^{\circ} \mathrm{C}\right)$ with primary antibody. Primary antibody was removed, the membrane washed for $6 \times 10 \mathrm{~min}$, and Horse Radish Peroxidase (HRP) conjugated secondary antibody added for $1 \mathrm{~h}$ (RT). The membrane was washed $(6 \times 10 \mathrm{~min})$ and bands visualised using Chemiluminescent HRP Substrate (Millipore). Antibodies used were rat anti-MLKL [1] (1/1000; clone $3 \mathrm{H} 1$, available as cat. MABC604 from Millipore), rabbit anti-GAPDH (1/1000; glyceraldehyde-3-phosphate dehydrogenase; Cell Signalling Technology), mouse antiVDAC1 (1/500; Voltage Dependent Anion Channel 1; Merck, cat. MABN504) and mouse anti-actin (1/3000; Sigma).

\section{Statistical analyses}

Cell death assay results are presented as a mean \pm s.e.m. of at least three independent experiments (each conducted with a minimum of two biologically independent replicates for MDFs).

Acknowledgements We thank staff at the Australian Synchrotron SAXS/WAXS beamline for assistance with data collection. We acknowledge support from an AINSE Postgraduate Research Award and an Australian Government Research Training Program Scholarship for KAD; a Victorian International Research Scholarship to MCT; RQ was supported by a scholarship from the Walter and Eliza Hall Institute as part of the International Student Program in Research Experience. We are grateful to the National Health and Medical Research Council for fellowship (PEC, 1079700; JS, 1058190; JMM, 1105754), grant (1057905; 1124735) and infrastructure (IRIISS 9000433) support; the Australian Research Council (Future Fellowship to MDWG, FT140100544); and to the Victorian Government Operational Infrastructure Support scheme.

\section{Compliance with ethical standards}

Conflict of interest J.M.M., J.S., P.E.C., E.J.P. and S.N.Y. contribute to a project funded by Anaxis Pharma to develop necroptosis inhibitors. The remaining authors declare that they have no conflict of interest.

\section{References}

1. Murphy JM, Czabotar PE, Hildebrand JM, Lucet IS, Zhang JG, Alvarez-Diaz S, et al. The pseudokinase MLKL mediates necroptosis via a molecular switch mechanism. Immunity. 2013;39:443-53.

2. Sun L, Wang H, Wang Z, He S, Chen S, Liao D, et al. Mixed lineage kinase domain-like protein mediates necrosis signaling downstream of RIP3 kinase. Cell. 2012;148:213-27.

3. Wu J, Huang Z, Ren J, Zhang Z, He P, Li Y, et al. Mlkl knockout mice demonstrate the indispensable role of Mlkl in necroptosis. Cell Res. 2013;23:994-1006.

4. Zhao J, Jitkaew S, Cai Z, Choksi S, Li Q, Luo J, et al. Mixed lineage kinase domain-like is a key receptor interacting protein 3 downstream component of TNF-induced necrosis. Proc Natl Acad Sci USA. 2012;109:5322-7.

5. Silke J, Rickard JA, Gerlic M. The diverse role of RIP kinases in necroptosis and inflammation. Nat Immunol. 2015;16:689-97.

6. Rickard JA, O'Donnell JA, Evans JM, Lalaoui N, Poh AR, Rogers TW, et al. RIPK1 regulates RIPK3-MLKL driven systemic inflammation and emergency hematopoiesis. Cell. 2014; 157:1175-88.

7. Rickard JA, Anderton H, Etemadi N, Nachbur U, Darding M, Peltzer N, et al. TNFR1-dependent cell death drives inflammation in Sharpin-deficient mice. Elife 2014;3:e03464.

8. Dannappel M, Vlantis K, Kumari S, Polykratis A, Kim C, Wachsmuth L, et al. RIPK1 maintains epithelial homeostasis by inhibiting apoptosis and necroptosis. Nature. 2014;513:90-4.

9. Degterev A, Huang Z, Boyce M, Li Y, Jagtap P, Mizushima N, et al. Chemical inhibitor of nonapoptotic cell death with therapeutic potential for ischemic brain injury. Nat Chem Biol. 2005;1:112-9.

10. Linkermann A, Brasen JH, Darding M, Jin MK, Sanz AB, Heller $\mathrm{JO}$, et al. Two independent pathways of regulated necrosis mediate ischemia-reperfusion injury. Proc Natl Acad Sci USA. 2013;110:12024-9.

11. Muller T, Dewitz C, Schmitz J, Schroder AS, Brasen JH, Stockwell BR, et al. Necroptosis and ferroptosis are alternative cell death pathways that operate in acute kidney failure. Cell Mol Life Sci. 2017;74:3631-45.

12. Luedde M, Lutz M, Carter N, Sosna J, Jacoby C, Vucur M, et al. RIP3, a kinase promoting necroptotic cell death, mediates adverse remodelling after myocardial infarction. Cardiovasc Res. 2014;103:206-16.

13. Gautheron J, Vucur M, Reisinger F, Cardenas DV, Roderburg C, Koppe C, et al. A positive feedback loop between RIP3 and JNK controls non-alcoholic steatohepatitis. EMBO Mol Med. 2014;6:1062-74.

14. Gunther C, He GW, Kremer AE, Murphy JM, Petrie EJ, Amann $\mathrm{K}$, et al. The pseudokinase MLKL mediates programmed hepatocellular necrosis independently of RIPK3 during hepatitis. J Clin Invest. 2016;126:4346-60.

15. Wang H, Sun L, Su L, Rizo J, Liu L, Wang LF, et al. Mixed lineage kinase domain-like protein MLKL causes necrotic membrane disruption upon phosphorylation by RIP3. Mol Cell. 2014;54:133-46.

16. Li J, McQuade T, Siemer AB, Napetschnig J, Moriwaki K, Hsiao YS, et al. The RIP1/RIP3 necrosome forms a functional amyloid signaling complex required for programmed necrosis. Cell. 2012;150:339-50.

17. Newton K, Wickliffe KE, Maltzman A, Dugger DL, Strasser A, Pham VC, et al. RIPK1 inhibits ZBP1-driven necroptosis during development. Nature. 2016;540:129-33.

18. Rodriguez DA, Weinlich R, Brown S, Guy C, Fitzgerald P, Dillon $\mathrm{CP}$, et al. Characterization of RIPK3-mediated phosphorylation of 
the activation loop of MLKL during necroptosis. Cell Death Differ. 2016;23:76-88.

19. Tanzer MC, Tripaydonis A, Webb AI, Young SN, Varghese LN, Hall C, et al. Necroptosis signalling is tuned by phosphorylation of MLKL residues outside the pseudokinase domain activation loop. Biochem J. 2015;471:255-65.

20. Cook WD, Moujalled DM, Ralph TJ, Lock P, Young SN, Murphy JM, et al. RIPK1- and RIPK3-induced cell death mode is determined by target availability. Cell Death Differ. 2014;21:1600-12.

21. Cai Z, Jitkaew S, Zhao J, Chiang HC, Choksi S, Liu J, et al. Plasma membrane translocation of trimerized MLKL protein is required for TNF-induced necroptosis. Nat Cell Biol. 2014;16:55-65.

22. Chen X, Li W, Ren J, Huang D, He WT, Song Y, et al. Translocation of mixed lineage kinase domain-like protein to plasma membrane leads to necrotic cell death. Cell Res. 2014;24:105-21.

23. Hildebrand JM, Tanzer MC, Lucet IS, Young SN, Spall SK, Sharma P, et al. Activation of the pseudokinase MLKL unleashes the four-helix bundle domain to induce membrane localization and necroptotic cell death. Proc Natl Acad Sci USA. 2014;111:15072-7.

24. Quarato G, Guy CS, Grace CR, Llambi F, Nourse A, Rodriguez DA, et al. Sequential engagement of distinct MLKL phosphatidylinositol-binding sites executes necroptosis. Mol Cell. 2016;61:589-601.

25. Dondelinger Y, Declercq W, Montessuit S, Roelandt R, Goncalves A, Bruggeman I, et al. MLKL compromises plasma membrane integrity by binding to phosphatidylinositol phosphates. Cell Rep. 2014;7:971-81.

26. Tanzer MC, Matti I, Hildebrand JM, Young SN, Wardak A, Tripaydonis A, et al. Evolutionary divergence of the necroptosis effector MLKL. Cell Death Differ. 2016;23:1185-97.

27. Huang D, Zheng X, Wang ZA, Chen X, He WT, Zhang Y, et al. The MLKL channel in necroptosis is an octamer formed by tetramers in a dyadic process. Mol Cell Biol. 2017;37:5.

28. Petrie EJ, Hildebrand JM, Murphy JM. Insane in the membrane: a structural perspective of MLKL function in necroptosis. Immunol Cell Biol. 2017;95:152-9.

29. Murphy JM, Lucet IS, Hildebrand JM, Tanzer MC, Young SN, Sharma $\mathrm{P}$, et al. Insights into the evolution of divergent nucleotide-binding mechanisms among pseudokinases revealed by crystal structures of human and mouse MLKL. Biochem J. 2014;457:369-77.

30. Su L, Quade B, Wang H, Sun L, Wang X, Rizo J. A plug release mechanism for membrane permeation by MLKL. Structure. 2014;22:1489-1500.

31. Jacobsen AV, Lowes KN, Tanzer MC, Lucet IS, Hildebrand JM, Petrie EJ, et al. HSP90 activity is required for MLKL oligomerisation and membrane translocation and the induction of necroptotic cell death. Cell Death Dis. 2016;7:e2051.

32. Brumatti G, Ma C, Lalaoui N, Nguyen NY, Navarro M, Tanzer MC, et al. The caspase- 8 inhibitor emricasan combines with the SMAC mimetic birinapant to induce necroptosis and treat acute myeloid leukemia. Sci Transl Med. 2016;8:339ra369.

33. Murphy JM, Vince JE. Post-translational control of RIPK3 and MLKL mediated necroptotic cell death. F1000Res. 2015;4:F1000.

34. Chen W, Zhou Z, Li L, Zhong CQ, Zheng X, Wu X, et al. Diverse sequence determinants control human and mouse receptor interacting protein 3 (RIP3) and mixed lineage kinase domain-like (MLKL) interaction in necroptotic signaling. J Biol Chem. 2013;288:16247-61.

35. Bigenzahn JW, Fauster A, Rebsamen M, Kandasamy RK, Scorzoni S, Vladimer GI, et al. An inducible retroviral expression system for tandem affinity purification mass-spectrometry-based proteomics identifies mixed lineage kinase domain-like protein (MLKL) as an heat shock protein 90 (HSP90) client. Mol Cell Proteom. 2016;15:1139-50.

36. Zhao XM, Chen Z, Zhao JB, Zhang PP, Pu YF, Jiang SH, et al. Hsp90 modulates the stability of MLKL and is required for TNFinduced necroptosis. Cell Death Dis. 2016;7:e2089.

37. Murphy JM, Metcalf D, Young IG, Hilton DJ. A convenient method for preparation of an engineered mouse interleukin-3 analog with high solubility and wild-type bioactivity. Growth Factors. 2010;28:104-10.

38. Hercus TR, Barry EF, Dottore M, McClure BJ, Webb AI, Lopez $\mathrm{AF}$, et al. High yield production of a soluble human interleukin-3 variant from E. coli with wild-type bioactivity and improved radiolabeling properties. PLoS ONE. 2013;8:e74376.

39. Schuck P. Size-distribution analysis of macromolecules by sedimentation velocity ultracentrifugation and lamm equation modeling. Biophys J. 2000;78:1606-19.

40. Laue TM, Shah, BD, Ridgeway, TM \& Pelletier, SL Analytical ultracentrifugation in biochemistry and polymer science. In: SE Harding AJRJCH (ed). Royal Society of Chemistry, 1992.

41. Kirby NM, Mudie ST, Hawley AM, Cookson DJ, Mertens HDT, Cowieson $\mathrm{N}$, et al. A low-background-intensity focusing smallangle X-ray scattering undulator beamline. J Appl Crystallogr. 2013;46:1670-80.

42. Konarev PV, Volkov VV, Sokolova AV, Koch MHJ, Svergun DI. PRIMUS: A Windows PC-based system for small-angle scattering data analysis. J Appl Crystallogr. 2003;36:1277-82.

43. Svergun DI. Determination of the regularization parameter in indirect-transform methods using perceptual criteria. J Appl Crystallogr. 1992;25:495-503.

44. Petoukhov MV, Svergun DI. Global rigid body modeling of macromolecular complexes against small-angle scattering data. Biophys J. 2005;89:1237-50. 